\title{
Hydrophobic Recovery in Ultrathin PDMS-coated Long and Short Silicon Nanowires
}

D. Ganta ${ }^{2,3^{*}}$ C. Marry ${ }^{1}$, J. Ma ${ }^{1}$, S. Sinha ${ }^{1,2}$

${ }^{1}$ Department of Mechanical Science and Engineering, University of Illinois, Urbana, Illinois 61801, USA

${ }^{2}$ Micro and Nanotechnology Laboratory, University of Illinois, Urbana, Illinois 61801, USA

${ }^{3}$ School of Engineering, Texas A\&M International University, Laredo, Texas 78041, USA

*Corresponding author.

E-mail address: deepak.ganta@tamiu.edu.

The ability to modify the contact angle (CA) of water on silicon has applications ranging from thermal management of electronics to miniaturized biomedical devices. Here, we report $\sim 30 \pm 1^{\circ}$ variation in superhydrophobic $\mathrm{CA}$ on silicon nanowires (NWs) coated with few $\mathrm{nm}$ of polydimethylsiloxane (PDMS), using a simple and stable simple plasma treatment. The variation in CA arises from choosing NWs of different lengths. We characterize the surfaces using a combination of X-ray photoelectron spectroscopy and other techniques. Together with CA available from similar treatment on bulk silicon, it is possible to non-lithographically create regions of diverse $\mathrm{CA}$, from $\sim 5$ to $149 \pm 1^{\circ}$.

KEYWORDS: silicon, superhydrophobic, contact angle, PDMS recovery, plasma oxidation, nanowires 


\section{INTRODUCTION}

The inherent hydrophobicity or hydrophilicity of a surface is determined by both its chemical makeup and surface texture. Control of wettability on silicon in particular is of interest in a wide range of applications. Bare silicon is hydrophilic and can be rendered superhydrophyllic after micro- or nano-structuring [1]. Surface treatments can also modify the wetting characteristic to hydrophobic behavior. The latter is attractive in a host of biological applications. For example, in micro-contact printing where PDMS microfluidic channels can be patterned with biological ink such as proteins or DNA [2-4]. Hydrophilic/hydrophobic surfaces are also interesting for energy conversion systems and thermal management [5].

Several surface treatments can alter the surface of bulk PDMS, especially oxygen plasma, UV, and silanization [2, 6-11]. PDMS is naturally hydrophobic before plasma treatment or reactive ion etching (RIE). On exposure to oxygen plasma, PDMS switches from hydrophobic to hydrophilic. Oxygen atoms attach to methyl group locations, forming $\mathrm{OH}$ groups at the surface of the PDMS leading to a silica-like layer [6-8]. However, bulk PDMS exposed to oxygen plasma recovers hydrophobicity within a few hours, unless it is immersed in water [6]. The recovery has been studied extensively [2,11-15]. The leading argument is that the low molecular weight groups (LMWGs) from the bulk move up along the silica layer while $\mathrm{OH}$ groups at the surface rearrange into the bulk of the PDMS, completely restoring the hydrophobicity with time [2, 6, 16-19]. The recovery is within six hours, and rules out applications requiring long-term hydrophobicity. Obtaining a long-term permanent reduction in the hydrophobicity of PDMS is more complicated. One example is a plasma treatment involving a combination of several gases operating at $100 \mathrm{~W}$, where the range of water contact angle (CA) is $60-85 \pm 1^{\circ}$ [2]. This is more suited for DNA and proteins micro-contact printing applications where the suitable water CA window is $50-90 \pm 1^{\circ}[2-4]$. The motivation for this work is to explore simpler oxygen plasma treatments that can both expand the overall range of contact angles and ensure long-term behavior.

In this paper, we explore a relatively simple method to obtain a wide range of contact angles on silicon based on coating a nm-scale PDMS film ( 3- $6 \mathrm{~nm})$ on either silicon or silicon nanowires using vapor deposition followed by short ( 5 seconds) exposure to oxygen plasma operating at low power $(13 \mathrm{~W})$. Scaling down the thickness of PDMS to a few nm does not effect bio- 
compatibility for microprinting applications [20]. The extent of recovery after the plasma treatment depends on the thickness of the PDMS layer. Varying the thickness within a narrow range yields a short range of CA. In order to further increase the range of possible contact angles after recovery, we introduce nanoscale roughness via NWs on the silicon substrate. After coating with $\sim 3 \mathrm{~nm}$ of PDMS, these surfaces become superhydrophobic $\left(\mathrm{CA}>150^{\circ}\right)$. We track and compare changes in the degree of hydrophobicity of different samples over an extended period of several months. CA measurements are supported by a detailed analysis of the surface chemistry and thickness changes for both coated and uncoated samples, using high resolution Xray photoelectron spectroscopy (XPS) along with an analysis for NWs.

\section{METHODS}

PDMS Coating Procedure. An elastomer base and a curing agent (Corning Sylgard 184) were thoroughly mixed in a 10:1 mass ratio in a petri dish to obtain roughly 10-mm thickness of PDMS. Bubbles were then removed by placing petri dish in a desiccator connected to the vacuum line. Both Si and Si NW samples were then coated with PDMS using a simple vapor deposition technique. The sample along with a piece of a PDMS stamp are placed in a sealed glass container and heated at $235^{\circ} \mathrm{C}$ for 30 minutes to obtain $\sim 3 \mathrm{~nm}$ thick layer.

Plasma Treatment. After coating, Si and Si NW samples were exposed to oxygen plasma treatment or RIE. The exposure took place in a Plasma Therm chamber with a base pressure of 20 mTorr, oxygen flow rate of 19.6 SCCM, and RF plasma power of $13 \mathrm{~W}$ at various exposure times (5-50 seconds). The PDMS coated samples are then stored in ambient conditions.

Fabrication of Nanowire Arrays. RCA-1 cleaned (100) silicon wafers $(1-10 \Omega \mathrm{cm})$ were used for the fabrication. The first step is e-beam evaporation of $\sim 10 \mathrm{~nm}$ of silver (Ag) film, maintaining a pressure of $10^{-6}$ Torr in the vacuum chamber during deposition. Dewetting process by rapid thermal annealing the sample at $350^{\circ} \mathrm{C}$ was done right after $\mathrm{Ag}$ deposition in order to avoid degradation of Ag layer. Ag layer turns into Ag droplets used as a mask for depositing gold. Gold mesh is then formed by e-beam evaporation of $\sim 10 \mathrm{~nm} \mathrm{Au}$, followed by lift-off of Ag particles by placing the sample in an Ag etch solution and sonicating for 15 minutes. Gold is used as a catalyst for the MACE process, followed by top-down chemical etching in a $12 \mathrm{~g}$ : $1.7 \mathrm{~g}: 8.4 \mathrm{~g}$ solution of $[\mathrm{HF}]:\left[\mathrm{H}_{2} \mathrm{O}_{2}\right]$ : [Ethanol] with a noble metal $(\mathrm{Au})$ as a 
catalyst to generate vertically aligned Si NWs. The sample was than rinsed in ethanol and DI water, followed by removal of Au using a gold etching solution for $\sim 3$ minutes.

Characterization. XPS was performed using a Kratos Axis-165 tool (monochromatic Al-K $\alpha$ X-ray source $15 \mathrm{kV}, 10 \mathrm{~mA}$, and $90^{\circ}$ ) for element analysis and to understand the details of surface chemistry. Survey scans were acquired followed by high-resolution scans at $40 \mathrm{eV}$ with a charge- neutralization system running during the analysis. We used $\mathrm{C} 1 \mathrm{~s}$ as reference for peak shifts during charging, which was almost negligible $(\sim 1 \mathrm{eV})$ due to a charge-neutralization system. The high resolution scans were peak fitted using CASA XPS software and baselines set to Shirley-type background. A Hitachi S-4800 field emission scanning electron microscope (SEM) was used for imaging at 10-30 KV. We measured the thickness of PDMS using an ellipsometry (Gaertner L116C) technique assuming refractive index of PDMS to be 1.46.

Contact Angle Measurement. The wetting properties of the samples are measured on a regular basis using a Rame-Hart contact angle goniometer that has a CCD camera for image capture. The images of water droplet are processed to obtain a contact angle.

\section{RESULTS AND DISCUSSION}

A simple, lithography-free metal-assisted chemical etching (MACE) process is used to fabricate nanowires on a Si substrate [21]. We characterized the NWs using scanning electron microscopy (SEM). Figure 1 (a) shows the SEM image of the cross-section of the nanowire array (top and side views). The image is used to determine the diameter (50-150) nm and length $(\sim 1.8 \mu \mathrm{m})$ of nanowires, and yields a rough estimation of the roughness $(\sim 120 \mathrm{~nm})$ through an image processing software (ImageJ). Similarly SEM image of the top view of the longer NW array is shown in Figure 1 (b). As the length of the NW increases clumping among the NWs increases. This is evident from comparing the top view SEM images of both short and long NWs. We can control the length of the NWs by varying the etching time inside the solution of [HF]: $\left[\mathrm{H}_{2} \mathrm{O}_{2}\right.$ ]: [Ethanol] ( 11 nm/second), with $\pm 5 \%$ deviation in the dimensions after the growth.

Vapor deposition was used to coat the nanowires with $\sim 3 \mathrm{~nm}$ of PDMS. The thickness was approximately controlled by varying the time of exposure to PDMS vapor [22]. We employed similar vapor deposition settings to coat Si and Si NW samples. The PDMS sample along with $\mathrm{Si} / \mathrm{Si} \mathrm{NW}$ sample is placed in a covered glass container and heated at $235^{\circ} \mathrm{C}$ to 
convert to a vapor phase. Increasing the heating time in 15 minute intervals increased the thickness by $\sim 1-2 \mathrm{~nm}$. By varying the exposure time we can control thickness of PDMS film from $~ 3-6 \mathrm{~nm}$ with 5-10\% deviation. After heat treatment, the short PDMS chains formed on the surface further crosslink immediately, resulting in the formation of a thin PDMS coating [22].

The thickness of PDMS deposited on Si substrate was measured using ellipsometry and verified further using X-ray photoelectron spectroscopy (XPS). We note that high-resolution transmission electron microscopy (HR-TEM) could not conclusively distinguish the thickness of the native oxide from that of the PDMS in the case of Si NW samples coated with PDMS. Using the standard two layer model for a flat surface in XPS, the expression for thickness (d) of the PDMS film on a silicon substrate, is given by[23]:

$$
\mathrm{d}=\lambda \ln \left(\frac{c_{a}}{c_{b}}+1\right) \sin \theta
$$

where $\mathrm{c}_{a}$ is the overlayer atomic concentration, $\mathrm{c}_{b}$ is the substrate atomic concentration, $\theta$ is the takeoff angle $\left(90^{\circ}\right)$ relative to the surface, and $\lambda$ is the inelastic photo-electron mean free path. The mean free path is $\sim 3 \mathrm{~nm}$ [23]. Both concentrations were obtained by analyzing the XPS spectra for carbon. For a silicon sample coated with a target thickness of $\sim 3 \mathrm{~nm}$ PDMS via vapor deposition, the XPS analysis yielded a thickness of $\sim 3.2 \mathrm{~nm}$. Ellipsometry measured the thickness to be $\sim 2.7 \mathrm{~nm}$.

XPS was further used to investigate changes in surface chemistry for Si and Si NW samples coated with PDMS before and after exposure to oxygen plasma. A survey scan is the first step in the XPS analysis to determine the elements present on the surface. This is followed by a highresolution XPS scan for detailed analysis of the elements of interest. Figure 2 shows a survey scan for a silicon surface coated with $\sim 3 \mathrm{~nm}$ PDMS. The survey scan shows C 1 s peak at 284.3 $\mathrm{eV}, \mathrm{O} 1 \mathrm{~s}$ peak at $532 \mathrm{eV}$ and $\mathrm{Si} 2 \mathrm{p}$ at $100 \mathrm{eV}$. High resolution XPS spectra are used to study the surface changes for the following samples: bare Si, Si coated with $\sim 3 \mathrm{~nm}$ thin PDMS, Si coated with $\sim 3 \mathrm{~nm}$ thin PDMS immediately after 5 seconds and 50 seconds of oxygen plasma treatment or reactive ion etching (RIE), and Si NWs coated with $\sim 3.0 \mathrm{~nm}$ PDMS with 5 sec plasma treatment. Any sample exposed to air will have adventitious carbon detectable by XPS. Since 
the PDMS thickness of our samples is within the penetration depth of the XPS beam $(\sim 8 \mathrm{~nm})$, the $\mathrm{C}$ detected is a combination of both adventitious C and C from DMS (dimethylsiloxane), molecules of PDMS as seen in Figure 3(a).

Figure 3(a) shows the overlay of XPS spectra on the same scale of intensity (a.u), before and after $\sim 3 \mathrm{~nm}$ PDMS coating. The main difference is in the peak intensity of $\mathrm{C} 1 \mathrm{~s}$ due to the attachment of dangling bonds of $\mathrm{C}$ in the DMS molecules on the Si surface. This is in a vapor state immediately after PDMS coating. Figure 3(b) is the high resolution $\mathrm{C}$ 1s peak after the exposure to oxygen plasma. The weak C-C peak arises in both treated and untreated PDMS samples. Figure 3(c) is the overlay of $\mathrm{C} 1$ s peak intensity of short $(\sim 1.8 \mu \mathrm{m})$ and long $(\sim 27 \mu \mathrm{m})$ Si NW samples 2-3 months after RIE, both of which are coated with $\sim 3 \mathrm{~nm}$ thin PDMS. In the subsequent text, "short" nanowires will refer to $\sim 2 \mu \mathrm{m}$ long wires and "long" nanowires will refer to $\sim 20 \mu \mathrm{m}$ long wires.

Table 1 compares the surface chemical composition changes (At \%) for C 1s, O 1s and Si 2p between bare Si, Si coated with $~ 3 \mathrm{~nm}$ PDMS, and Si coated with $\sim 3 \mathrm{~nm}$ PDMS immediately after $5 \mathrm{sec}$ and $50 \mathrm{sec}$ RIE. The results point towards the formation of a silica-like layer $\left(\mathrm{SiO}_{\mathrm{x}}\right)$ after exposure to oxygen plasma and the origin of it is already predicted and discussed in the literature $[2,17,18,19]$. This is evident from the increase in $\mathrm{Si}$ and $\mathrm{O}$ with respect to $\mathrm{C}$. The sample with 50 seconds of exposure to oxygen plasma shows less $\mathrm{C}$ content, indicating the removal of PDMS or larger cracks. Table 1 also reports data for uncoated NWs as well as PDMS coated short and long NWs.

Figure 4 (a) and (b) shows high resolution Si 2p XPS scans for a bare silicon sample and bare NW sample. The nanowires are short enough for XPS to observe the bulk Si substrate, so the Si $2 \mathrm{p}$ peaks look similar for all the cases. The two peaks of the $\mathrm{Si} 2 \mathrm{p}$ region correspond to elemental silicon $\left(\mathrm{Si}^{0}\right)$ and silicon dioxide $\left(\mathrm{SiO}_{2}\right)$, at $100 \mathrm{eV}$ and $103.8 \mathrm{eV}$ respectively, the intensity of which varies as the sample is exposed to oxygen plasma. The silicon dioxide peak for the bare silicon sample indicates the presence of a native oxide on the silicon wafer. The NW sample has a stronger $\mathrm{SiO}_{2}$ peak than elemental silicon because the surface area exposed to oxygen under ambient conditions is greater for the NW sample compared to the bare silicon sample. 
After exposure to oxygen plasma, all coated samples were regularly monitored for 3 months until the final CA value stabilized. Three different thicknesses of PDMS-coated silicon were tested: $\sim 3 \mathrm{~nm}, \sim 4.5 \mathrm{~nm}$, and $\sim 6 \mathrm{~nm}$. Each thickness was tested after 5 second oxygen plasma exposure times. We measure the CA within 10 minutes after the plasma exposure. Figure 5 shows the change in CA over time for $3 \mathrm{~nm}, 4.5 \mathrm{~nm}$ and $6 \mathrm{~nm}$ PDMS coated silicon wafers after 5 second oxygen plasma exposure. The CA was measured at 5 different locations on each sample. The reported values are each averaged over 5 measurements. The error bars in Figure 5 show standard deviation calculated for each set of CA measurements on each sample. The standard deviation from the reported values in water CA is $\pm 1^{\circ}$. Before RIE, all of the coated Si samples had CA between $90-100^{\circ}$, which is normal for bulk PDMS. After RIE, the samples exhibited lower CA that however increased over the course of several weeks but never reached their original values. Images of water droplets used in the CA measurements at each step of the process are shown in Figure 6(a)-(d). The sample in this case is silicon NWs coated with $\sim 3 \mathrm{~nm}$ PDMS.

The $\sim 3 \mathrm{~nm}$ thick PDMS-coated bulk Si samples that had been exposed for $5 \mathrm{sec}$ recovered to $47 \pm 1^{\circ}$ after a few months and stayed stable as shown in Figure 5. This is likely due to the fact that $\mathrm{nm}$ thin layer of PDMS have very few low molecular weight groups left after oxidation to aid in the recovery process. The $4.5 \mathrm{~nm}$ and $6 \mathrm{~nm}$ coated bulk samples with 5 seconds of RIE recovered to approximately $65 \pm 1^{\circ}$ after a period of 2-3 months. We suspect that for thicknesses less than $\sim 3 \mathrm{~nm}$ the hydrophobicity might never recover because all of the PDMS would be oxidized or etched.

Long Si NWs with an initial $\left(\mathrm{CA}<5 \pm 1^{\circ}\right.$ ) when coated with $\sim 3 \mathrm{~nm}$ of PDMS exhibited superhydrophobicity with CA of $157.14 \pm 1^{\circ}$. Immediately after RIE, the hydrophobicity reduced to $121.78 \pm 1^{\circ}$. After tracking the sample for several months, we found partial hydrophobic recovery to a CA of $149 \pm 1^{\circ}$ as shown in Figure 5. The same study was repeated for shorter NWs S1 ( 1.8 micron long) with initial CA of $150 \pm 1^{\circ}$, after RIE the final value stabilized at $117 \pm 1^{\circ}$ with no recovery, as shown in Figure 5. The experiment was repeated for another set of NWs S2 ( 2 microns long) which had CA of $15 \pm 1^{\circ}$ after PDMS coating. Immediately after RIE, the CA was $101 \pm 1^{\circ}$ and recovered after 2 weeks to $118 \pm 1^{\circ}$. 
The final value of the CA post the recovery process for both Si and Si NW samples coated with PDMS, varied between $\left(47-149 \pm 1^{\circ}\right)$ as shown in Table 2, when tracked over a period of 2-3 months. The effect of the $5 \mathrm{sec}$ RIE exposure for various thicknesses of PDMS is also summarized in Table 2. As discussed above, the primary reason why hydrophobicity is not completely restored for $\mathrm{Si} / \mathrm{Si} \mathrm{NW}$ samples coated with thin PDMS is due to not many low molecular weight groups left to aid the complete recovery process.

While the variation in the final contact angle for bulk Si is understandable, the behavior of NWs is puzzling. There is significant difference $\left(\sim 30 \pm 1^{\circ}\right)$ in the final CA between short and long NWs. From previous studies, the intrinsic length of the NWs does not have any significant effect on the dewetting properties of the surface beyond $100 \mathrm{~nm}$ [24-26]. The contact angle of uncoated nanowire array is however affected by the spacing between the nanowires [24]. During fabrication, nanowires that are taller than $\sim 1.3 \mu \mathrm{m}$ tend to form clusters with increasingly larger clusters as the length increases. This is evident from the top view SEM images shown in Figure 1. Long Si NWs are more tilted than the short NWs, as illustrated in Figure 1. There were difference observed in MACE grown Si NWs in roughness morphology on the surface and the crystal orientation, some of them being smooth and others being slightly rough as length of the NWs increases [27, 28]. Vertical one's were shown to be smoother compared with titled ones, which were more porous. It is more difficult to uniformly coat the exposed surface of freestanding wires that are not clustered since the mean free path of the plasma is comparable to the spacing between the wires. Once clustered, the spacing between the clusters is large enough for the plasma to coat the clusters on the outside. We suspect that the shorter NW sample did not recover completely due to the presence of thinner PDMS coating compared with longer NWs. This is also seen in Figure 3(c) and Table 1 when comparing the variation in $\mathrm{C}$ (At\%) between the samples. We note that the $\sim 30^{\circ}$ variation in CA that we observe in our nm-thin PDMS coated NW samples is an order of magnitude larger than that previously observed [24] in uncoated clustered NW samples where the variation is within $6^{\circ}$. Thus, using ultrathin PDMS coatings in conjunction with NWs enables a wider range of CAs.

Simply varying the thickness and/or roughness on different areas on the same Si surface, the technique we report hold promise for single-cell transfer printing of various molecules on the same surface. 


\section{CONCLUSION}

In summary, we observe that the presence of few nanometers thick PDMS on silicon followed by RIE yields CA between $47-65 \pm 1^{\circ}$ that are relatively stable after a period of 2-3 months post an initial recovery. The range of CA is determined by the variation in the thickness of PDMS. Using this technique, hydrophilic PDMS stamps can be used for microcontact printing of DNA and protein and stored over longer periods of time without need for immersing in water or for a more invasive method of surface modification involving polar groups. Similarly we can also reduce the superhydrophobicity of intrinsic Si NW surfaces from $\sim 150-155 \pm 1^{\circ}$ to a range within $117-149 \pm 1^{\circ}$ using nanometer-thin PDMS. The variation in CA arises from choosing NWs of different lengths and is approximately five times more than that observed previously due to clustering effects alone. This has potential applications for patterning bio-markers such as gold nanoparticles and in waste heat management.

Acknowledgement : This work was supported in part by the U.S. Navy through Grant No. N66001-11-1-4154 and in part by the National Science Foundation through Grant No. NSFCBET-12-50192. We would like to thank Richard Haasck for assisting with XPS measurements. 


\section{REFERENCES}

[1] T.S. Wong, C.M. Ho, Dependence of Macroscopic Wetting on Nanoscopic Surface Textures, Langmuir, 25 (2009) 12851-12854.

[2] B.J. Larson, S.D. Gillmor, J.M. Braun, L.E. Cruz-Barba, D.E. Savage, F.S. Denes, M.G. Lagally, Long-Term Reduction in Poly(dimethylsiloxane) Surface Hydrophobicity via ColdPlasma Treatments, Langmuir, 29 (2013) 12990-12996.

[3] G. Piret, Y. Coffinier, C. Roux, O. Melnyk, R. Boukherroub, Biomolecule and nanoparticle transfer on patterned and heterogeneously wetted superhydrophobic silicon nanowire surfaces, Langmuir, 24 (2008) 1670-1672.

[4] E. Delamarche, C. Donzel, F.S. Kamounah, H. Wolf, M. Geissler, R. Stutz, P. SchmidtWinkel, B. Michel, H.J. Mathieu, K. Schaumburg, Microcontact printing using poly(dimethylsiloxane) stamps hydrophilized by poly(ethylene oxide) silanes, Langmuir, 19 (2003) 8749-8758.

[5] R. Chen, M.C. Lu, V. Srinivasan, Z. Wang, H.H. Cho, A. Majumdar, Nanowires for Enhanced Boiling Heat Transfer, Nano Lett, 9 (2009) 548-553.

[6] J.L. Fritz, M.J. Owen, Hydrophobic recovery of plasma-treated polydimethylsiloxane, J Adhesion, 54 (1995) 33-45.

[7] M.J. Owen, P.J. Smith, Plasma Treatment of Polydimethylsiloxane, J Adhes Sci Technol, 8 (1994) 1063-1075.

[8] Y.Z. Wu, Y.Y. Huang, H.W. Ma, A facile method for permanent and functional surface modification of poly(dimethylsiloxane), J Am Chem Soc, 129 (2007) 7226-+.

[9] K. Ma, J. Rivera, G.J. Hirasaki, S.L. Biswal, Wettability control and patterning of PDMS using UV-ozone and water immersion, J Colloid Interf Sci, 363 (2011) 371-378.

[10] E.A. Waddell, S. Shreeves, H. Carrell, C. Perry, B.A. Reid, J. McKee, Surface modification of Sylgard 184 polydimethylsiloxane by $254 \mathrm{~nm}$ excimer radiation and characterization by contact angle goniometry, infrared spectroscopy, atomic force and scanning electron microscopy, Appl Surf Sci, 254 (2008) 5314-5318.

[11] D.T. Eddington, J.P. Puccinelli, D.J. Beebe, Thermal aging and reduced hydrophobic recovery of polydimethylsiloxane, Sensor Actuat B-Chem, 114 (2006) 170-172.

[12] N.J. Peter, X.S. Zhang, S.G. Chu, F.Y. Zhu, H. Seidel, H.X. Zhang, Tunable wetting behavior of nanostructured poly(dimethylsiloxane) by plasma combination treatments, Appl Phys Lett, 101 (2012).

[13] H. Hillborg, N. Tomczak, A. Olah, H. Schonherr, G.J. Vancso, Nanoscale hydrophobic recovery: A chemical force microscopy study of UV/ozone-treated cross-linked poly(dimethylsiloxane), Langmuir, 20 (2004) 785-794. 
[14] R.A. Lawton, C.R. Price, A.F. Runge, W.J. Doherty, S.S. Saavedra, Air plasma treatment of submicron thick PDMS polymer films: effect of oxidation time and storage conditions, Colloid Surface A, 253 (2005) 213-215.

[15] S. Bhattacharya, A. Datta, J.M. Berg, S. Gangopadhyay, Studies on surface wettability of poly(dimethyl) siloxane (PDMS) and glass under oxygen-plasma treatment and correlation with bond strength, J Microelectromech S, 14 (2005) 590-597.

[16] H. Hillborg, U.W. Gedde, Hydrophobicity recovery of polydimethylsiloxane after exposure to corona discharges, Polymer, 39 (1998) 1991-1998.

[17] H. Hillborg, S. Karlsson, U.W. Gedde, Characterisation of low molar mass siloxanes extracted from crosslinked polydimethylsiloxanes exposed to corona discharges, Polymer, 42 (2001) 8883-8889.

[18] H. Hillborg, M. Sandelin, U.W. Gedde, Hydrophobic recovery of polydimethylsiloxane after exposure to partial discharges as a function of crosslink density, Polymer, 42 (2001) 73497362.

[19] J. Kim, M.K. Chaudhury, M.J. Owen, Modeling hydrophobic recovery of electrically discharged polydimethylsiloxane elastomers, J Colloid Interf Sci, 293 (2006) 364-375.

[20] K.Y. Chumbimuni-Torres, R.E. Coronado, A.M. Mfuh, C. Castro-Guerrero, M.F. Silva, G.R. Negrete, R. Bizios, C.D. Garcia, Adsorption of proteins to thin-films of PDMS and its effect on the adhesion of human endothelial cells, Rsc Adv, 1 (2011) 706-714.

[21] X.L. Li, Metal assisted chemical etching for high aspect ratio nanostructures: A review of characteristics and applications in photovoltaics, Curr Opin Solid St M, 16 (2012) 71-81.

[22] J.K. Yuan, X.G. Liu, O. Akbulut, J.Q. Hu, S.L. Suib, J. Kong, F. Stellacci, Superwetting nanowire membranes for selective absorption, Nat Nanotechnol, 3 (2008) 332-336.

[23] K.R. Finnie, R. Haasch, R.G. Nuzzo, Formation and patterning of self-assembled monolayers derived from long-chain organosilicon amphiphiles and their use as templates in materials microfabrication, Langmuir, 16 (2000) 6968-6976.

[24] M.K. Dawood, H. Zheng, N.A. Kurniawan, K.C. Leong, Y.L. Foo, R. Rajagopalan, S.A. Khan, W.K. Choi, Modulation of surface wettability of superhydrophobic substrates using Si nanowire arrays and capillary-force-induced nanocohesion, Soft Matter, 8 (2012) 3549-3557.

[25] Y.H. Cheng, C.K. Chou, C. Chih, S.Y. Cheng, Critical length of nanowires for hydrophobic behavior, Chem Phys Lett, 397 (2004) 17-20.

[26] W.F. Kuan, L.J. Chen, The preparation of superhydrophobic surfaces of hierarchical silicon nanowire structures, Nanotechnology, 20 (2009) 035605.

[27] W. Chern, K. Hsu, I.S. Chun, B.P.d. Azeredo, N. Ahmed, K.-H. Kim, J.-m. Zuo, N. Fang, P. Ferreira, X. Li, Nonlithographic Patterning and Metal-Assisted Chemical Etching for Manufacturing of Tunable Light-Emitting Silicon Nanowire Arrays, Nano Lett, 10 (2010) 15821588.

[28] H. Han, Z. Huang, W. Lee, Metal-assisted chemical etching of silicon and nanotechnology applications, Nano Today, 9 (2014) 271-304. 
(a)
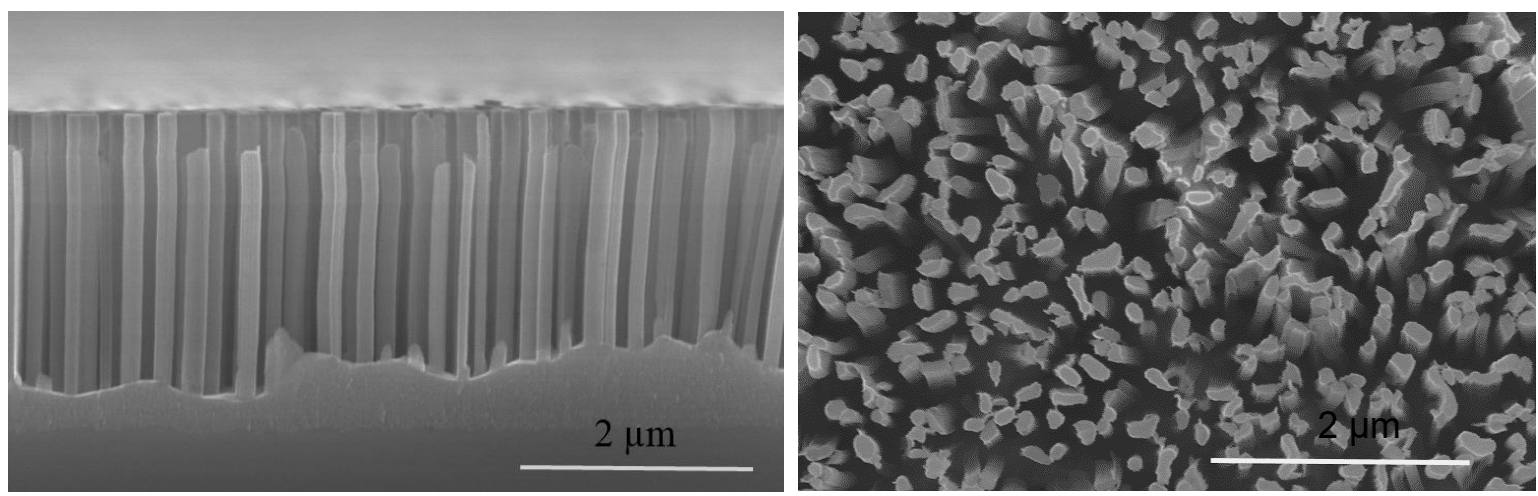

(b)

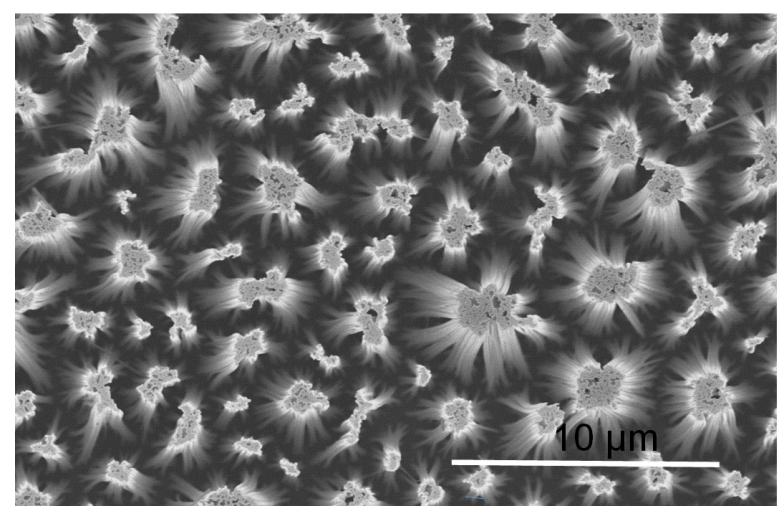

Figure 1: SEM image of the cross section of. (a) Short NW (left) side view and (right) top view, and (b) Top view of a long NW. 


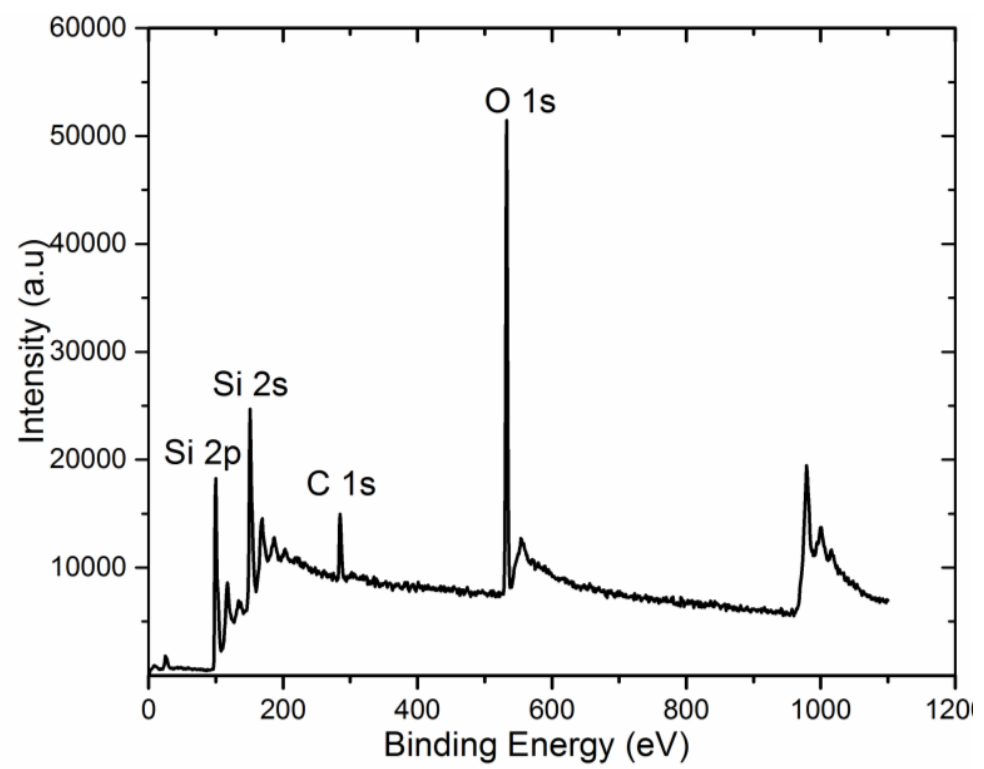

Figure 2: The survey XPS spectra for silicon substrate coated with $\sim 3 \mathrm{~nm}$ PDMS film. 
(a)

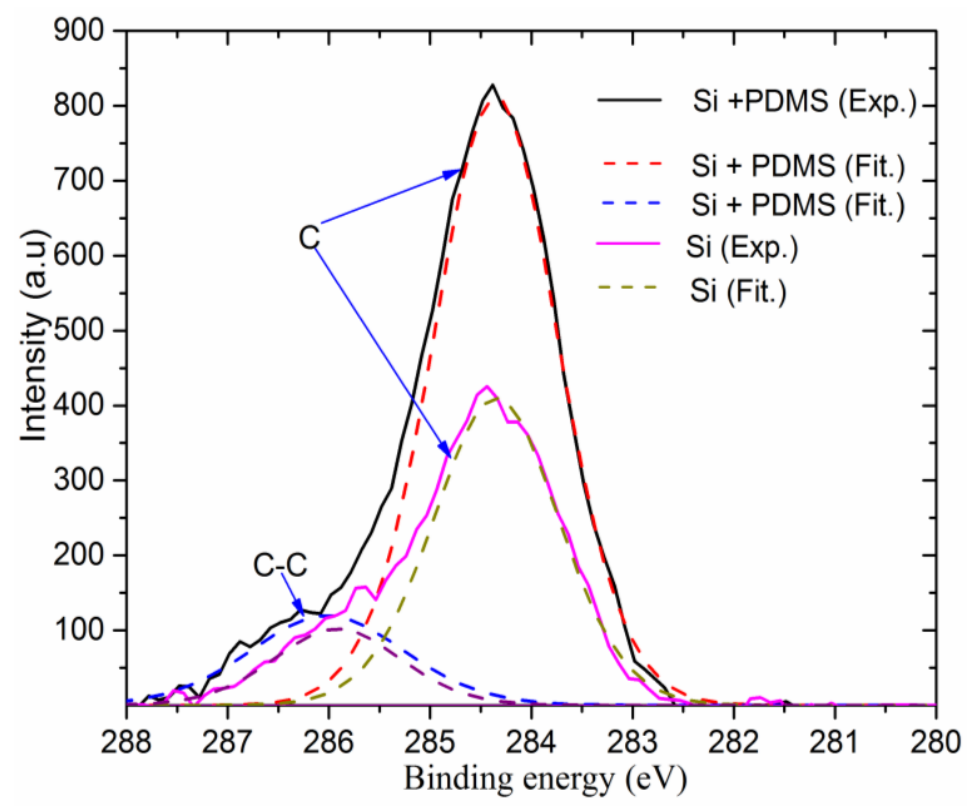

(b)

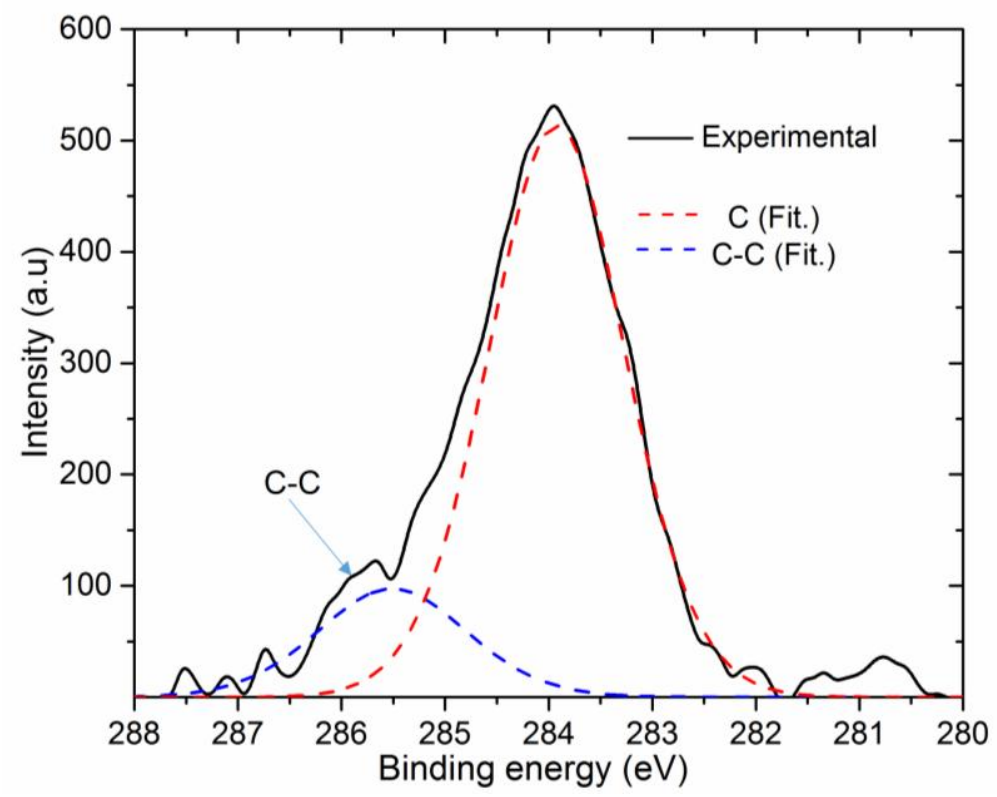


(c)

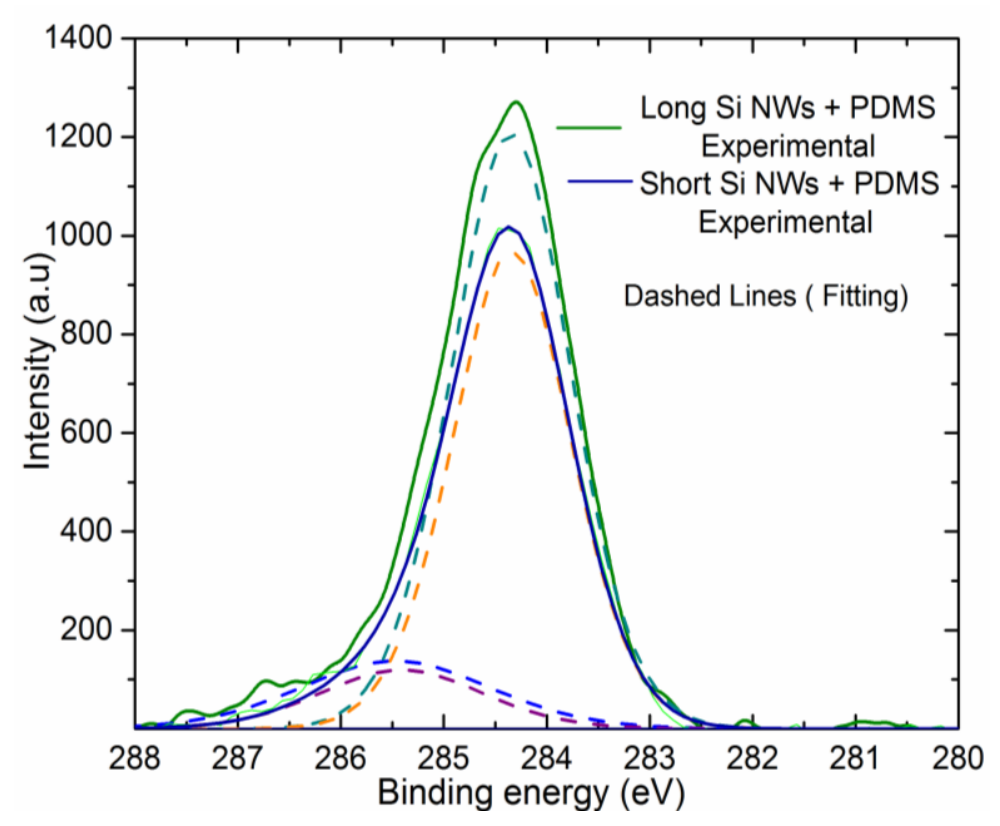

Figure 3: High resolution XPS spectra of the C 1s region: (a) Overlay of bare Si sample vs. Si coated with $\sim 3 \mathrm{~nm}$ of PDMS. (b) PDMS coated Si sample immediately after oxygen plasma RIE for 5 seconds. (c) Overlay of Si NWs $(1.8 \mu \mathrm{m}$ vs. $28 \mu \mathrm{m})$, coated with $\sim 3 \mathrm{~nm}$ PDMS. Solid Lines indicate experimental data and dashed lines are curve fitting from CASA XPS software. 
(a)

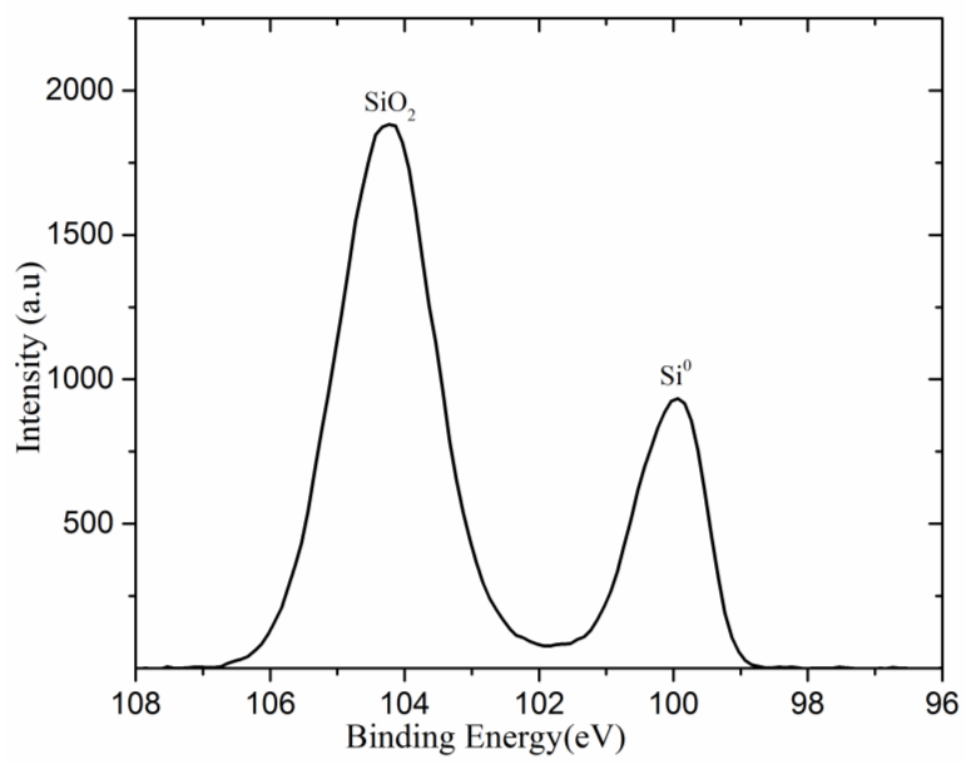

(b)

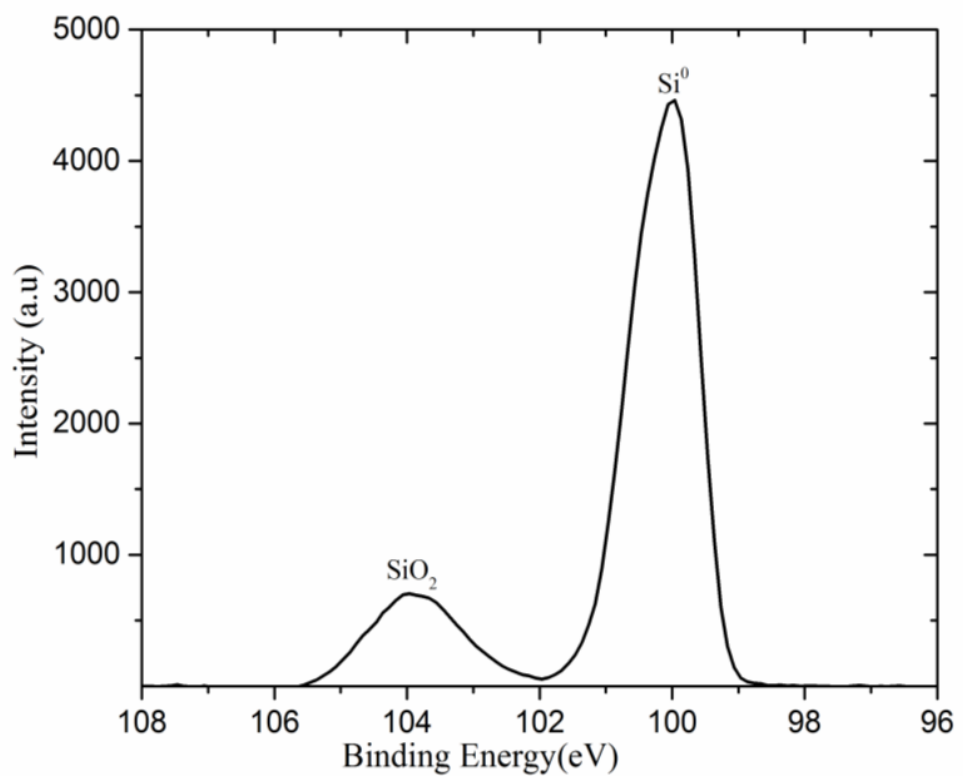

Figure 4: High resolution Si 2p XPS spectra of: (a) Bare silicon substrate, and (b) Silicon Nanowire. 


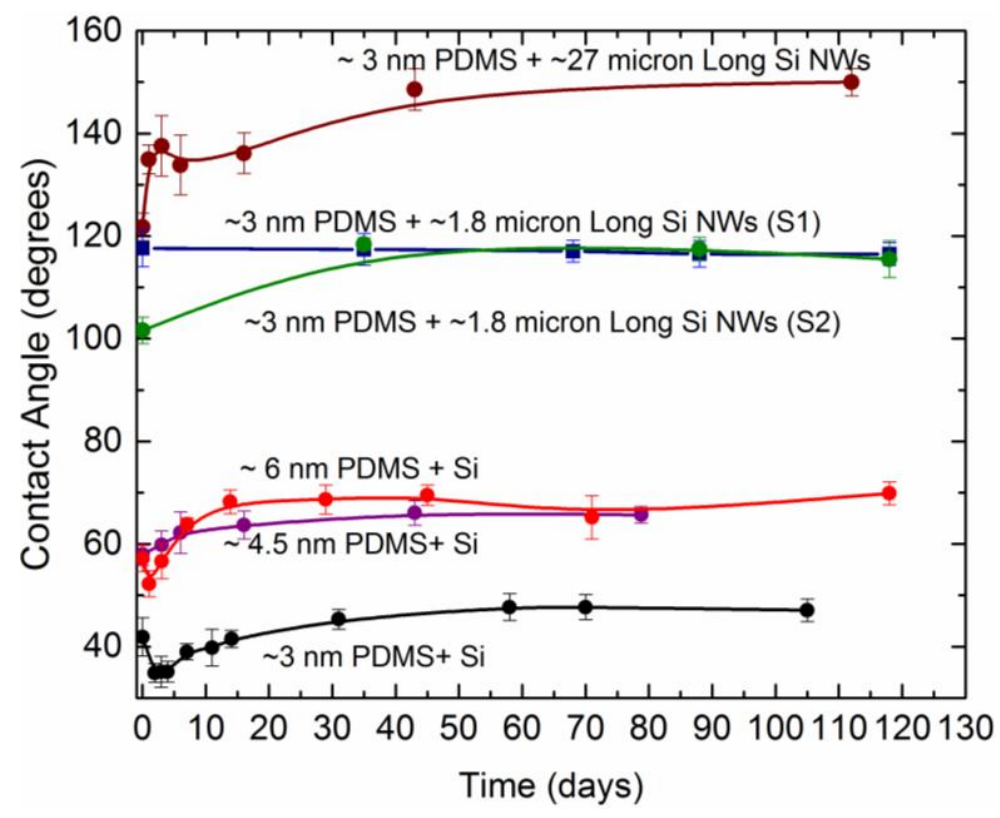

Figure 5. Evolution of water contact angle vs. time for PDMS coated Si/Si NW samples; after 5 sec oxygen plasma exposure, for various thicknesses of PDMS and dimensions of NWs. 


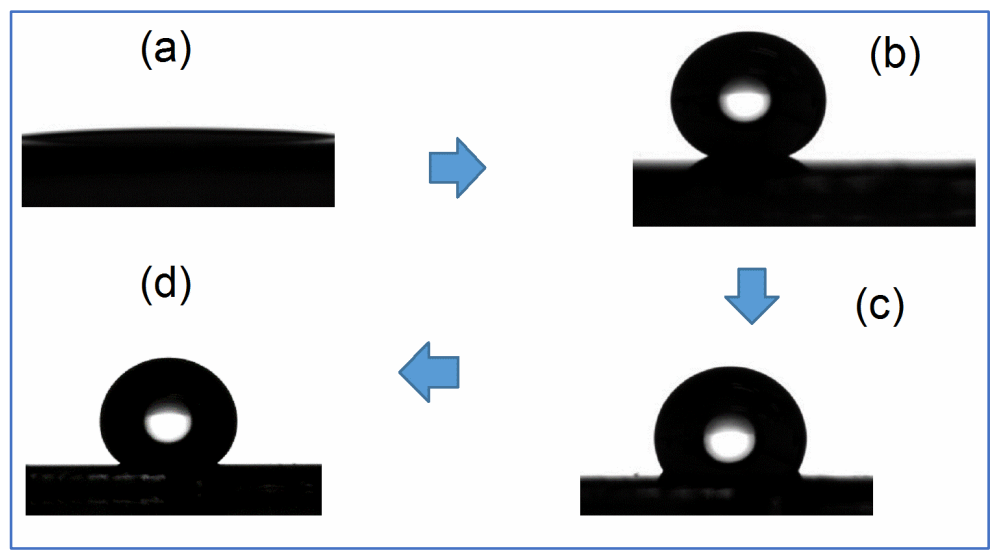

Figure 6. Evolution of CA of water droplet with time. (a) As- made Si NWs, (b) Long Si NWs coated with $\sim 3$ nm PDMS, (c) PDMS coated NWs; immediately after plasma treatment, and (d) PDMS coated NWs; 115 days after plasma treatment. 
Table 1. Composition before and after oxygen plasma treatment.

\begin{tabular}{|c|c|c|c|c|c|c|c|}
\hline & (At \%) & $\begin{array}{l}\mathrm{Si}+\sim 3 \mathrm{~nm} \\
\mathrm{PDMS} \\
\text { (At \%) }\end{array}$ & $\begin{array}{l}\mathrm{Si}+\sim 3 \mathrm{~nm} \\
\text { PDMS } \\
\text { after } 5 \mathrm{sec} \\
\text { RIE } \\
(\text { At \%) }\end{array}$ & $\begin{array}{l}\mathrm{Si}+\sim 3 \mathrm{~nm} \\
\text { PDMS } \\
\text { after } 50 \mathrm{sec} \\
\text { RIE } \\
(\text { At \%) }\end{array}$ & $($ At $\%)$ & $\begin{array}{l}\text { Long Si } \\
\text { NWs + } \\
\text { PDMS } \\
\text { after RIE } \\
(\text { At } \%)\end{array}$ & $\begin{array}{l}\text { Short Si } \\
\text { NWs + } \\
\text { PDMS } \\
\text { after RIE } \\
(\text { At \%) }\end{array}$ \\
\hline C 1s & 7.60 & 14.72 & 5.60 & 3.51 & 10.95 & 18.41 & 13.70 \\
\hline Si $2 p$ & 65.15 & 57.45 & 60.74 & 62.82 & 42.96 & 40.51 & 44.54 \\
\hline $\mathrm{O} 1 \mathrm{~s}$ & 27.24 & 27.82 & 33.66 & 33.68 & 46.09 & 41.76 & 41.76 \\
\hline
\end{tabular}


Table 2: CA measurements after 5 second oxygen plasma treatment.

\begin{tabular}{|c|c|c|c|c|c|}
\hline Sample & $\begin{array}{l}\text { Target } \\
\text { thickness } \\
(\mathrm{nm})\end{array}$ & $\begin{array}{l}\text { CA before } \\
\text { PDMS } \\
\text { coating } \\
\text { (deg) }\end{array}$ & $\begin{array}{l}\text { CA after } \\
\text { PDMS } \\
\text { coating } \\
(\text { deg })\end{array}$ & $\begin{array}{l}\text { CA immediately } \\
\text { after RIE } \\
\text { (deg) }\end{array}$ & $\begin{array}{l}\text { CA after } \\
90 \text { days } \\
\text { post RIE } \\
\text { (deg) }\end{array}$ \\
\hline Bare Si & 3 & 45.00 & 90.96 & 41.90 & 47.70 \\
\hline Bare Si & 4.5 & 45.00 & 100.04 & 57.96 & $65.70^{*}$ \\
\hline Bare Si & 6 & 45.00 & 96.37 & 57.15 & 65.19 \\
\hline Si NWs $(27 \mu \mathrm{m})$ & 3 & 5.00 & 157.14 & 121.78 & 149.02 \\
\hline $\begin{array}{lll}\mathrm{Si} & \mathrm{NWs} & (1.8 \\
\mu \mathrm{m}) & (\mathrm{S} 1)\end{array}$ & 3 & 5.00 & 150.00 & 117.00 & 117.00 \\
\hline $\begin{array}{lll}\mathrm{Si} & \mathrm{NWs} & (1.8 \\
\mu \mathrm{m}) & (\mathrm{S} 2) & \\
\end{array}$ & 3 & 5.00 & 151.00 & 101.00 & 118.00 \\
\hline
\end{tabular}

* CA after 80 days.

$\underline{\text { Note: Standard deviation in water CA values are } \pm 1^{\circ}}$ 

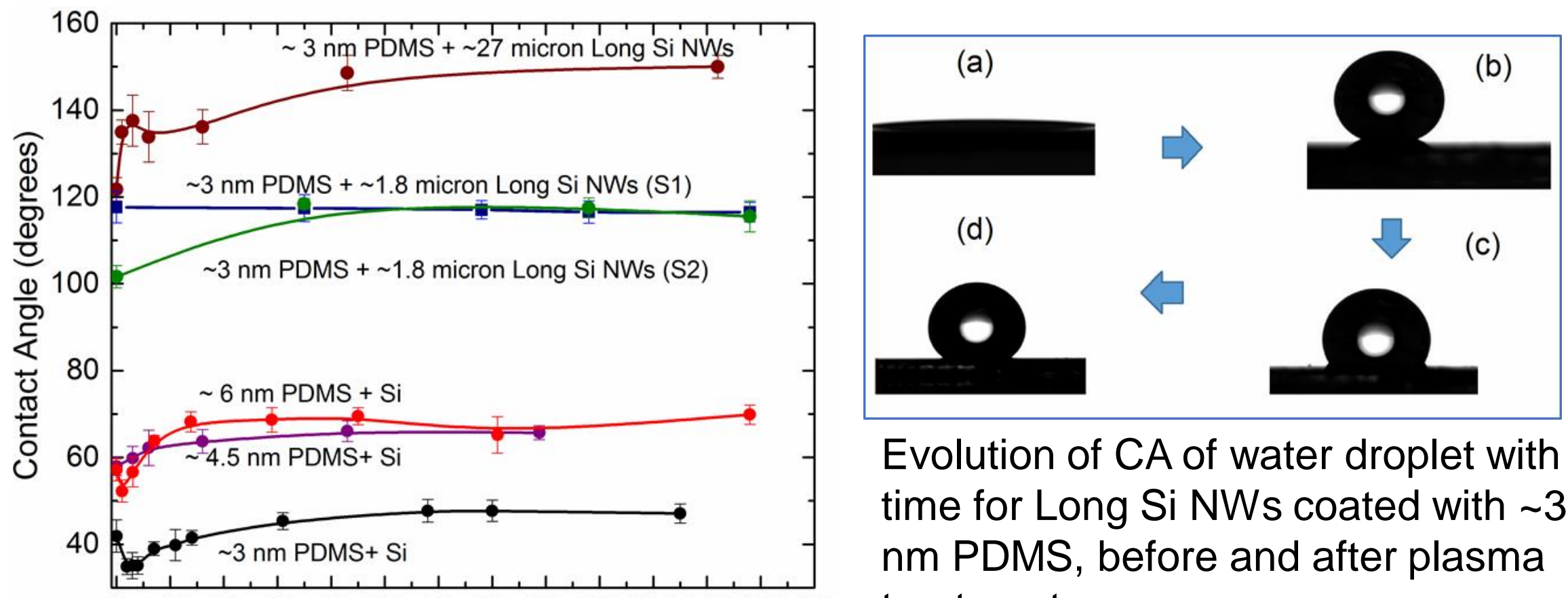

Evolution of CA of water droplet with time for Long Si NWs coated with $\sim 3$ $\mathrm{nm}$ PDMS, before and after plasma treatment

Time (days) 